\title{
An Irreversible Heat Engine Working at the Reversible Efficiency
}

\author{
José C. Íñiguez \\ Correspondence: José C. Íñiguez, Retired instructor of science and mathematics, USA. E-mail: iniguez.jose@gmail.com
}

Received: June 2, 2020 Accepted: June 27, 2020 Online Published: July 2, 2020

doi:10.5539/ijc.v12n2p1 URL: https://doi.org/10.5539/ijc.v12n2p1

\begin{abstract}
The thermodynamic analysis of the coupling of one cycle in the operation of a reversible heat engine with a workdegrading step in which the whole of the engine's work output is frictionally degraded into heat at the temperature of its cold reservoir, allows identification of the fact that the engine's reversibility is dependent on the continued availability of its work output. As long as this work remains available the engine will be reversible, this on reason of the fact that the initial condition can be restored via the simple expedient of using the said work to propel the inverse cycle. The moment this work becomes, for whatever reason, unavailable, restoration of the engine's initial condition becomes impossible, and what was a reversible engine becomes irreversible. The inability of current thermodynamic terminology to deal with this situation is brought to light and a simple suggestion aimed at correcting this deficiency is advanced.
\end{abstract}

Keywords: restoration of the initial condition, reversibility, optimal efficiency, work availability, reversibility degree

\section{Reversibility}

According to Planck:

A process which can in no way be completely reversed is termed irreversible, all other processes reversible. That a process may be irreversible, it is not sufficient that it cannot be directly reversed...The full requirement is, that it be impossible, even with the assistance of all agents in nature, to restore everywhere the exact initial state when the process has once taken place. (Planck 1960/1926, p. 84)

As noted in Planck's previous quote, it is the 'restoration everywhere of the exact initial state' what constitutes the requirement to be fulfilled if a process is to be called reversible. In other words, it is only on reason of the fact that all of the bodies involved in the process of interest can be returned to the precise condition each of them had before any change had taken place that the process can be called reversible.

Let us start recognizing that any process taking place in the absence of any dissipative agents such as conduction, friction, electrical resistance, etc. is an optimally efficient process. In the case of spontaneous processes this qualifier means maximum work output, while in those non-spontaneous it translates into minimum work consumption. Let us then consider the optimally efficient isothermal expansion of an ideal gas. Besides the gas, this process demands the concourse of two other bodies: a heat reservoir and a mechanical reservoir. In it the heat $Q$ transferred by the heat reservoir to the gas ends up being transformed, due to the said absence of dissipation, into an equivalent amount of work $W$ which ends up in the mechanical reservoir. If we retrieve now the work previously deposited in the mechanical reservoir and use it to perform, also in the absence of dissipation, the isothermal compression of the gas, we will find out not only that the amount of work $W$ made available by the expansion is precisely the amount required to push the gas back to its initial condition, but also that the amount of heat which originating in the spent work ends up being transferred to the heat reservoir, is identical to that this reservoir originally released to the gas. Once this forward/reverse combination of processes has come to an end we will see all the bodies involved returning to their respective initial conditions: 1) the gas because it was forced to do so; 2) the mechanical reservoir because along the compression it released an amount of work identical to the one it had previously received along the expansion; and 3) the heat reservoir because along the compression it recuperated the amount of heat it initially released along the expansion. The fact that restoration is possible qualifies the expansion as reversible. The previous argument illustrates the meaning of restoration being the basis of proof for reversibility. Confirmation of the former is required before the latter qualifier can be attached to any given process

\section{Work-Degrading and the Reversible to Irreversible Transition}

Planck's previous definition of reversibility as the possibility of restoring the exact initial condition once a process has taken place will here be used to identify the reversible or irreversible condition of the processes of Figure 1. 


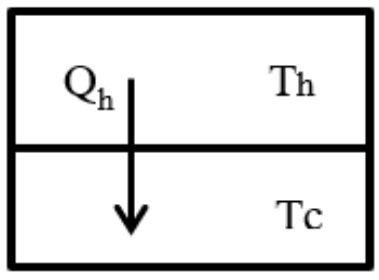

(a)

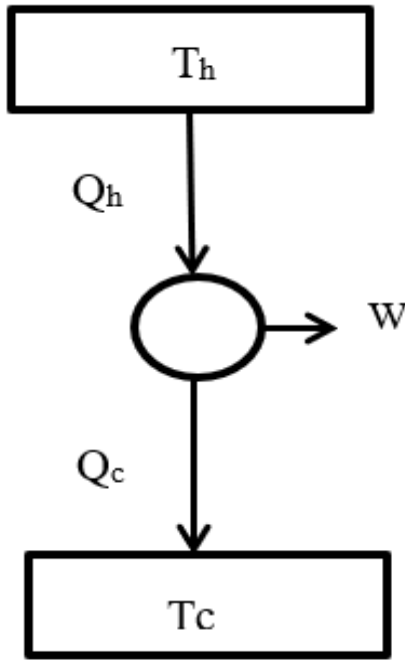

(b)

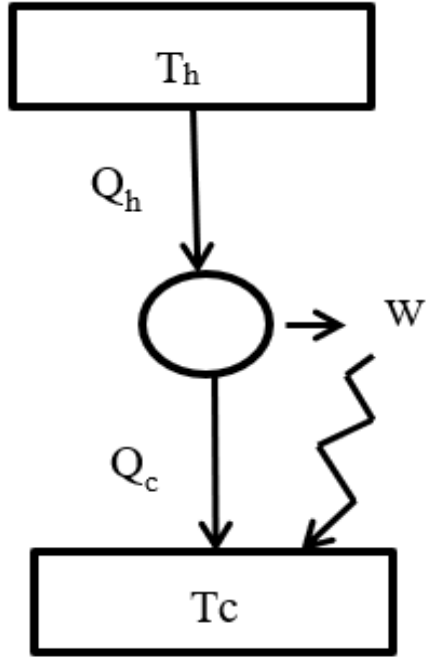

(c)

Figure 1. Two different paths to an irreversible heat transfer

Figure 1 In the figure above process (a) represents the direct, conductive transfer of an amount of heat $Q_{h}$ from a hot reservoir of temperature $T_{h}$ to a cold reservoir of temperature $T_{c}$. Process (b) represents one cycle in the operation of a reversible engine which out of the amount of heat $Q_{h}$ produces an amount of work $W=\eta_{\text {Carnot }} Q_{h}$, transferring the difference $Q_{c}=Q_{h}-W$ to a cold reservoir of temperature $T_{c}$. Process (c) is a representation of the result produced by the concatenation or combination of process (b) with a frictional step through which $W$ is degraded into an equivalent amount of heat at the temperature of the cold reservoir

Process (a) represents the direct, conductive transfer of an amount of heat $Q_{h}$ from a hot reservoir of temperature $T_{h}$ to a cold reservoir of temperature $T_{c}$. This process, characterized by its null work output, is an irreversible process on reason of the fact that the only way possible to restore the initial condition once the said heat transfer has taken place is represented by the unassisted or spontaneous flow of $Q_{h}$ from the cold to the hot reservoir. This possibility is, however, negated by" (that) statement of experimental truth" expressed as "Heat cannot pass spontaneously from a body of lower temperature to a body of higher temperature" (Schmidt, 1966, p. 92). Surely, $Q_{h}$ might be transferred back from the cold to the hot reservoir when an optimally efficient Carnot refrigerator is inserted between the reservoirs. To run this refrigerator we need, however, an amount of work of magnitude $w=Q_{h}\left[\left(T_{h}-T_{c}\right) / T_{c}\right]($ Fermi, 1956/1937, p. 44) that we don't have as none was generated during the occurrence of process (a). This problem can be solved by bringing in a work-supplying body. Even if this procedure allows for the transfer back of $Q_{h}$ from the cold to the hot reservoir it is incapable of restoring everywhere the exact initial condition, this on reason of the fact that it leaves two changes behind: A work-supplying body depleted of an amount of energy $w$, and a hot reservoir taking back an amount of heat $w$ in excess of that it originally released.

Figure (b) represents the effects of one cycle in the operation of a reversible heat engine. The temperatures of the hot and cold reservoirs are assumed respectively identical to those of process (a). Out of the amount of heat $Q_{h}$ received from the hot reservoir, this optimally efficient engine produces the maximum possible amount of work consistent with its operating conditions $Q_{h}, T_{h}$, and $T_{c}$, namely $W=Q_{h} \eta_{\text {Carnot }}=Q_{h}\left[\left(T_{h}-T_{c}\right) / T_{h}\right]$, and transfers the difference $Q_{c}=Q_{h}-W$ to the cold reservoir. The work output $W$ appears in the form of an increase in the potential energy of some mechanical reservoir. The reversible label attached to this engine's operation is a consequence of the fact that the initial condition can be restored by the simple expedient of using $W$ to drive the inverse cycle, i.e. to run the engine in reverse.

Irreversible process (c) is the one produced when process (b) is followed by, or concatenated with, a frictional work-degrading step through which the work generated in the former ends up as an equivalent amount of heat in the cold reservoir. To understand the irreversibility of process (c) let us recognize that four are the bodies taking part in the concatenation of processes there depicted: The working substance, the heat reservoirs, and the mechanical reservoir. The working substance in a heat engine, a role played by steam in most engines, by air in some others, or an ideal gas in most theoretical discussions- is, as known, the thermal bridge connecting the hot and cold reservoirs, and on reason of this and its volume changes, the origin of the work output of the engine. At the conclusion of the said work-degrading process two of those four bodies, namely the working substance and the mechanical reservoir are found in their respective initial conditions, and the other two, namely the heat 
reservoirs, in conditions different to the ones they originally had. That no change remains in the working substance finds explanation in the fact that it returns to its initial condition at the conclusion of each and every one of the engine's cycles of operation. The conclusion of process (b) is actually signaled by the return of the working substance to its initial condition. That no change remains in the mechanical reservoir finds explanation in the fact that an identical amount of work to that it received from engine (b) has been retrieved from it to be degraded as heat at the temperature of the cold reservoir. In regard to the hot reservoir it is at once seen that the only change by it experienced is the release in process (b) of an amount of heat $Q_{h}$. The cold reservoir experiences, on its part, two changes. The first one, clearly depicted in Figure (b), consists in it receiving an amount of heat $Q_{c}$ from the working substance, with the second change consisting in it receiving an additional amount of heat $W$ from the work-degrading step, for a total amount of heat $Q_{h}=Q_{c}+W$. The fact that the concatenation of processes depicted in (c) reduces to the transfer of $Q_{h}$ from the hot to the cold reservoir makes it identical in its effect to irreversible process (a), and just like in process (a), restoration of the initial condition in (c) demands, on reason of the absence of $W$ in the mechanical reservoir, the concourse of the impossible process represented by the spontaneous transit of $Q_{h}$ from the cold to the hot reservoir. The fact that the insertion of a reversible refrigerator between the heat reservoirs is incapable of producing complete reversion has already been explained above.

Before the work degrading step engine (b) was reversible. As long as $W$ is available in the mechanical reservoir all we have to do to restore the initial condition is retrieve it and use it to propel the inverse cycle. After the loss of $W$ via the frictional process, engine (b) is irreversible. According to these results the reversible condition of engine (b) requires the continuous availability of $W$ to guarantee the restoration of the initial condition. As soon as this work is no longer available the possibility of restoration of the initial condition, and with it the reversible condition of this engine, disappears.

The previous notion has already been reported and applied to a number of problems, though not the one herein dealt with, in a number of publications (Íñiguez \& Íñiguez 2004; Íñiguez 2011, 2013, 2014a, 2014b).

\section{Reversibility in Heat Engines Means Work-Availability and Optimal Efficiency}

The previous argument, even if conducted around an optimally efficient engine, stressed the connection between reversibility and work availability. The present argument will show that both: optimal efficiency and work availability need to be satisfied if a heat engine is to be called reversible.

Let us then us center our attention on some heat engine with operating conditions $Q_{h}, T_{h}$, and $T_{c}$ identical to those of the previous arguments, and let us assume that on reason of our failure to prevent direct flow of heat from the hot to the cold reservoir (Pitzer \& Brewer, p. 95, 1961), a portion of the heat released by the hot reservoir manages to reach the cold reservoir bypassing the work-producing path offered by the volume changes of the working substance, and that on reason of this we are left with a decreased engine's work output $W^{\prime}, W^{\prime}<W$, where $W$, as before noted, represents the maximum work output for such an engine i.e., $W=\eta_{\text {Carnot }} Q_{h}$. Let us now inquire about the effect to be produced when an optimally efficient refrigerator working between the same temperatures is propelled with this decreased amount of work. Fed with $W^{\prime}$ the refrigerator will be capable of extracting from the cold reservoir an amount of heat equal to $Q_{c}^{\prime}=W^{\prime}\left[T_{c} /\left(T_{h}-T_{c}\right)\right]$. The ratio between this expression and the one corresponding to the refrigerator being fed with the optimally efficient engine's work output $W$, namely $Q_{c}=W\left[T_{c} /\left(T_{h}-T_{c}\right)\right]$, takes the form $Q_{c}^{\prime} / Q_{c}=W^{\prime} / W$. The fact that $W^{\prime}<W$ implies $Q_{c}^{\prime}<Q_{c}$. Decreased heat extraction follows the feeding of the refrigerator with decreased amounts of work. The quotient of works appearing in the previous equation doubles, it should be noted, as a quotient of efficiencies i.e., $W^{\prime} / W=\left(\eta^{\prime} Q_{h}\right) /\left(\eta_{\text {Carnot }} Q_{h}\right)=\eta^{\prime} / \eta_{\text {Carnot }}$, with $\eta^{\prime}$ representing the efficiency of the engine under consideration. The limit values of the $W^{\prime} / W$ quotient: zero for the irreversible condition represented by $W^{\prime}=0$, and 1 for the optimally efficient one represented by $W^{\prime}=W$, suggests the introduction of the parameter to be here called the 'reversibility degree', defined as $\phi=W^{\prime} / W=\eta^{\prime} / \eta_{\text {Carnot }}$, to be used for the quantification of the fraction of the initial condition susceptible of restoration. Consider, for example, a heat engine working within the following operating conditions $(200 \mathrm{~J}, 600 \mathrm{~K}, 300 \mathrm{~K}, 0.2)$ given in the order $\left(Q_{h}, T_{h}, T_{c}, \eta^{\prime}\right)$. From this data it follows that $\eta_{\text {carnot }}=0.5, W=100 \mathrm{~J}, Q_{c}=100 \mathrm{~J}, W^{\prime}=40 \mathrm{~J}$ and $Q_{c, i r r}^{\prime}=Q_{h}-W^{\prime}=160 \mathrm{~J}$. Note that this last magnitude quantifies the heat discharged by this engine to the cold reservoir. This amount of heat $Q_{c, \text { irr }}^{\prime}$ is larger than $Q_{c}$ in precisely the difference $W-W^{\prime}=60 \mathrm{~J}$ corresponding to the amount of work this inefficient operation falls short of its maximum possible on reason of the above assumed direct flow of heat between the heat reservoirs; this difference is the so-called lost-work . Recognition of the fact that here the initial condition is represented by a hot reservoir with a shareable amount of heat of $200 \mathrm{~J}$ will allow us to understand why the reversibility degree of this engine amounts to $\phi=W^{\prime} / W=40 / 100=0.4$ or $40 \%$. This is so because the previously noted refrigerator fed with the $40 \mathrm{~J}$ of work outputted by this engine can extract from the cold reservoir an amount of heat equal to $Q_{c}^{\prime}=40[300 /(600-300]=40 \mathrm{~J}$. That this amount of heat, alongside the dissipated work in the amount of $40 \mathrm{~J}$, end up replenishing the hot reservoir with $80 \mathrm{~J}$ out of the originally $200 \mathrm{~J}$ by it released, means a $(80 / 200) \cdot 100=40 \%$ restoration of the initial condition. $40 \%$ reversible, it should be understood, means $60 \%$ irreversible, and any irreversibility whatsoever, however small, means failure of restoration of the exact initial condition. Reversibility for this engine, a condition represented by $\phi=1$, or, equivalently, by $W^{\prime}=W$, requires the engine to be optimally efficient as it is only in this case that the engine will output the precise amount of work, $W=100 \mathrm{~J}$, required by the refrigerator to extract 
from the cold reservoir an amount of heat $Q_{c}=W\left[T_{c} /\left(T_{h}-T_{c}\right)\right]=100(300 / 300)=100 \mathrm{~J}$ identical to that previously transferred by this optimally efficient engine to this reservoir, and if so, transfer back to the hot reservoir an amount of heat $Q_{c}+W=100+100=200 \mathrm{~J}$ identical to that originally released by the hot reservoir. For a spontaneous process to be called reversible two conditions are then to be met: optimal-efficiency and the permanence of its work output. Failure to comply with both of these conditions, be it on reason of inefficiency or of work unavailability, leads, inevitably, to irreversibility understood as the inability of recuperating the exact initial condition. For $0 \leq \phi<1$, or equivalently for $W^{\prime}<W$, the operation is irreversible whether or not the work output remains available. For $\phi=1$ the operation can be called reversible as long as $W$ remains available. This last statement, which can also be expressed by saying that optimal efficiency is not synonymous with reversibility, is further discussed in the following section.

It has to be acknowledged that the discussion just presented is a simple conceptual extension from that forwarded by Carnot nearly two centuries ago via the following succinct discussion which makes reference to the concatenation of an optimally efficient heat engine (no lost-work) with its inverse cycle, operations these taking place between two bodies $A$ and $B$ acting as hot and cold reservoirs, respectively, via the transfer between them of a given amount of caloric:

By our first operations there would have been at the same time production of motive power and transfer of caloric from the body $A$ to the body $B$. By the inverse operations there is at the same time expenditure of motive power and return of caloric from the body $B$ to the body $A$. But if we have acted in each case on the same quantity of vapor, if there is produced no loss either or motive power or caloric, the quantity of motive power produced in the first place will be equal to that which would have been expended in the second, and the quantity of caloric passed in the first case from the body $A$ to the body $B$ would be equal to the quantity which passes back again in the second from the body $B$ to the body $A$; so that an indefinite number of operations of this sort could be carried on without in the end having either produced motive power or transferred caloric from one body to the other...so that these two series of operations annul each other, after a fashion, one neutralizing the other (Carnot, 1960/1824, pp. 11, 19).

\section{Optimal Efficiency Is Not a Synonym of Reversibility}

When reference is made to a reversible engine what is usually meant is an optimally efficient engine, i.e. one operating with Carnot's or so-called reversible efficiency. Out of all the engines working between the same hot and cold reservoirs it is this engine the one that manages to transform into work the largest fraction of every unit of heat made available by its hot reservoir. The efficiency of any given engine, it should be recognized, is a property of the engine and if so independent, among other things, of the destiny or final use of its work output. In other words, an optimally-efficient engine will keep efficiently transforming heat into work oblivious to whether its work output is being accumulated in a mechanical reservoir, or degraded into heat at the temperature of its cold reservoir or diverted to some other use. But even if the engine's efficiency experiences no change on reason of the different work-destinies previously described, the condition of this engine indeed does. In this regard let us recognize the following scenarios, 1) when the work output is directed to and remains in the engine's associated mechanical reservoir, the engine is reversible. As already explained, the reason for this is that the exact initial condition can be recuperated by the simple expedient of retrieving the work available in the mechanical reservoir and feeding it to the inverse cycle, 2) If the work output of this optimally-efficient engine is for whatever reason not available then the engine is irreversible; this for the simple reason that there is no work with which to propel the inverse cycle. If we attempt to describe this situation using common thermodynamic terminology we might end up talking about an irreversible engine working at the reversible efficiency. The previous description, which on reason of its contradictory nature appears nonsensical, originates in the incorrect position of making 'optimally-efficient' a synonym of 'reversible'. We have grown accustomed to take as certain the notion that any heat engine working at Carnot's efficiency is a reversible engine. As shown above, this identification is not always correct. As soon as the expression $\eta=\left(T_{h}-T_{c}\right) / T_{h}$ is referred to as Carnot's efficiency and any engine working at such efficiency as an optimally-efficient engine, the contradictory statement above presented could be favorably replaced by that referring to the irreversibility of an optimally-efficient engine.

\section{References}

Carnot, S. (1960/1824). Reflections on the Motive Power of Fire. Dover, NY.

Fermi, E. (1956/1937). Thermodynamics. Dover, NY.

Íñiguez, J. C. (2011). A thermodynamic impasse: A constant entropy irreversible process. Phys. Chem. Ind. J., 6(3), 125130. https://www.tsijournals.com/journals/archive/tspc-volume-6-issue-3-year-2011.html

Íñiguez, J. C. (2013). Infinite time irreversible processes. International Journal of Chemistry, 5(1), 20-25. https://doi.org/10.5539/ijc.v5n1p20

Íñiguez, J. C. (2014a). Work degrading and the reversible to irreversible transition in chemical reactions. International Journal of Chemistry, 6(3), 1-13. https://doi.org/10.5539/ijc.v6n3p1

Íñiguez, J. C. (2014b). Illustrating the connection between entropy production and lost work. Chemical Education Journal, 
Asian Edition, 16(1), 1-16. http://www.edu.utsunomiya-u.ac.jp/chem/v16n1/indexE.html

Íñiguez, J. C., \& Íñiguez, B. A. (2004). On thermodynamic reversibility. Chemistry Preprint Archive, $2004(3), 64-70$. https://papers.ssrn.com/abstract $=2978960$

Pitzer, K. S., \& Brewer, L. (1961). Thermodynamics. McGraw-Hill, Inc., NY.

Planck, M. (1990/1922). Treatise on Thermodynamics, Dover, NY.

Schmidt, E. (1966). Thermodynamics, Dover, NY.

\section{Copyrights}

Copyright for this article is retained by the author(s), with first publication rights granted to the journal.

This is an open-access article distributed under the terms and conditions of the Creative Commons Attribution license (http://creativecommons.org/licenses/by/4.0/). 